\title{
Photonic Angular Superresolution Using Twisted N00N States
}

\author{
Markus Hiekkamäki®, ${ }^{1, *}$ Frédéric Bouchard ${ }^{2}$ and Robert Fickler $\oplus^{1, \dagger}$ \\ ${ }^{1}$ Tampere University, Photonics Laboratory, Physics Unit, Tampere FI-33720, Finland \\ ${ }^{2}$ National Research Council of Canada, 100 Sussex Drive, Ottawa, Ontario K1A OR6, Canada
}

(Received 15 June 2021; revised 1 October 2021; accepted 16 November 2021; published 23 December 2021)

\begin{abstract}
The increased phase sensitivity of N00N states has been used in many experiments, often involving photon paths or polarization. Here we experimentally combine the phase sensitivity of N0ON states with the orbital angular momentum (OAM) of photons up to $100 \hbar$, to resolve rotations of a light field around its optical axis. The results show that both a higher photon number and larger OAM increase the resolution and achievable sensitivity. The presented method opens a viable path to unconditional angular supersensitivity and accessible generation of N00N states between any transverse light fields.
\end{abstract}

DOI: 10.1103/PhysRevLett.127.263601

During the past few decades, N00N states have been the focus of several studies where their potential was explored in different metrological applications [1-4]. Specifically, a N00N state refers to an extremal superposition of $N$ quanta between two orthogonal modes, i.e., $(1 / \sqrt{2})(|N, 0\rangle+$ $|0, N\rangle)$ [3]. These states owe their usefulness to an increased phase sensitivity that an $N$-photon Fock state has in comparison to a single photon, or more classical states of light. The increased phase sensitivity means that a phase $\phi$ affects the Fock state $|N\rangle N$ times, changing the state to $e^{i N \phi}|N\rangle$, whereas classical states of light would only gain the phase $\phi$ [3]. This increase in phase sensitivity has been utilized in many proof-of-principle experiments, most commonly by preparing two photons in a superposition of two paths [1] or polarizations [2]. One notable example is the demonstration of an unconditional quantum advantage in sensitivity, using two-photon polarization NO0N states [5].

Similarly to the phase sensitivity scaling with a photon number, the sensitivity in rotation measurements around the optical axis scales with the amount of helical twistedness in the wavefront of the light used [6]. This sensitivity is related to the rotational symmetry of the helically twisted wavefront of a light beam with nonzero orbital angular momentum (OAM). The amount of wavefront twistedness, or OAM, a photon can have is quantized to integer multiples $\ell$ of $\hbar$, and is theoretically unbounded [7], leading to a theoretically unbounded increase in measurement sensitivity. Experimentally, values of up to 10010 quanta of OAM

Published by the American Physical Society under the terms of the Creative Commons Attribution 4.0 International license. Further distribution of this work must maintain attribution to the author(s) and the published article's title, journal citation, and DOI. were already demonstrated [8]; however, this value is bounded by the finite aperture of the optical system [9].

Theoretical and experimental studies have examined methods of combining the increased phase sensitivity of quantum states and the optimal rotation sensitivity of light beams with large OAM [10-14]. In these studies however, instead of experimentally implemented twisted NOON states, the authors used either squeezed light states, light directly from a spontaneous parametric down conversion (SPDC) source, or multiple paths for the photons with different OAM values to travel. These implementations lack the robustness and simplicity of a single-path operation which can be achieved with the recently introduced method for bunching photons into different OAM NOON states [15].

In this study, we experimentally demonstrate an increased rotation sensitivity of twisted NOON states along a single path. With our method, we are able to show the increased rotation sensitivity using NOON states with photon numbers 1 and 2, and OAM values up to $100 \hbar$. Our results show that twisted two-photon N00N states have the potential for an angular uncertainty scaling $\propto(1 / \ell N)$, whereas classical light is limited to a scaling $\propto(1 / \ell \sqrt{N})[10,13]$. Although the amount of OAM is limited by the physical aperture, increasing the number of photons in a twisted NOON state has the potential to surpass any classical angular resolution limit. Due to the simplicity of the presented method, spatial mode N00N states with large OAM values and high efficiencies are achievable even with current technologies. As such, our work opens up novel ways to generate N00N states invoking the transverse spatial degree of freedom and offers a path to unconditional angular supersensitivity.

To create a two-photon twisted N00N state, two photons that have orthogonal transverse-spatial structures but are otherwise indistinguishable need to be brought into the same beam path. A unitary that transforms the modes into a 
mutually unbiased basis (MUB), i.e., a Hadamard operation $\hat{H}_{2}$, then leads to a bunching of the two photons into the original spatial structures [15]. This interference is analogous to the well-known Hong-Ou-Mandel interference realized by a beam splitter transformation [16]. However, since the beam-splitter-like transformation $\hat{H}_{2}$ is unitary, the two photons stay orthogonal in a certain basis

$$
\hat{H}_{2}|1,1\rangle_{\ell,-\ell}=\frac{1}{\sqrt{2}}\left(|2,0\rangle_{\ell,-\ell}-|0,2\rangle_{\ell,-\ell}\right)=|1,1\rangle_{M_{1}, M_{2}},
$$

where $M_{i}$ refer to the modes of another MUB of the OAM modes $\{\ell,-\ell\}$. Because of this feature, it is possible to include the beam-splitter-like operation into the state generation, before bringing the photons into the same beam path, while still achieving the same two-photon bunching and, thus, the same two-photon twisted NOON state. Interestingly, for larger photon numbers the process generates Holland-Burnett states that are also capable of overcoming the shot-noise limit [17].

To experimentally verify the efficacy of this method, we use an SPDC source to generate photon pairs and the setup shown in Fig. 1 (see Supplemental Material for details [18]). The photon pair is coupled out of single-mode fibers onto two separate regions of a spatial light modulator (SLM), as shown on the left side of Fig. 1, where the photons are structured using holographic phase and amplitude modulation $[19,20]$. The structured photons are then brought to the same path with a beam splitter to enable bunching into OAM structures. To measure the two-photon state, a second beam splitter probabilistically separates the photons, and a second SLM (SLM2) is used in conjunction with two single-mode fibers to filter the spatial structures of the photons independently [21,22]. Both of the SLMs that were used were wavefront corrected [23]. For singlephoton N00N states, only one input and output fiber were used, and the other photon was detected at the two-photon source, to herald a single-photon state [24].
To confirm that the photons bunch into a N00N state, we first prepare a two-photon NOON state with an OAM value of $\ell= \pm 1$, and verify its quantum correlations using an entanglement witness $[25,26]$. Measuring the state in all three MUBs, we achieve a witness value of $w=2.92 \pm 0.02$, which is greater than the maximal value of $w=1$ for separable states and close to the maximum value of $w=3$ of the witness for maximally entangled states.

After this initial confirmation, we proceed to examine the angular resolution and sensitivity of these OAM NOON states using our measurement scheme. In these measurements, we prepare heralded single photons and twophoton N00N states with OAM values $|\ell|=\{1,2,3,5$, $10,25,50,100\}$. For $|\ell|<10$, we use mode carving [19] and intensity flattening [21] to create and measure the structures, respectively. The amplitude modulation implemented in these procedures is needed to get as close as possible to the MUB states of OAM light fields. The OAM states have a complex field structure $E_{\ell}(\theta) \propto e^{i \ell \theta}$, where $\theta$ is the azimuthal coordinate. Hence, the MUB structures are of the form $E_{M_{1} / M_{2}}(\theta) \propto\left(e^{i \ell \theta} \pm e^{-i \ell \theta}\right)$ and are often called petal beams [27]. Examples of these structures for $\ell= \pm 2$ are shown in the insets of Fig. 1. When generating photons with $|\ell| \geq 10$, no amplitude modulation is required as the spatial structures are sufficiently filtered by the limited aperture of our system. Thus, simple phase imprinting [20] and phase flattening [22] are used to generate and measure the desired modes, respectively.

To demonstrate angular superresolution, we simulate the rotation of our photon structures by rotating the measurement holograms on SLM2. As with the more common twophoton Mach-Zehnder interferometer, a second Hadamard transformation is needed to detect the phase change. In the case of OAM modes, the second Hadamard transformation can be performed in the measurement by simply projecting the photons onto the petal mode basis (see Supplemental Material [18]). Hence, the two-photon state was measured

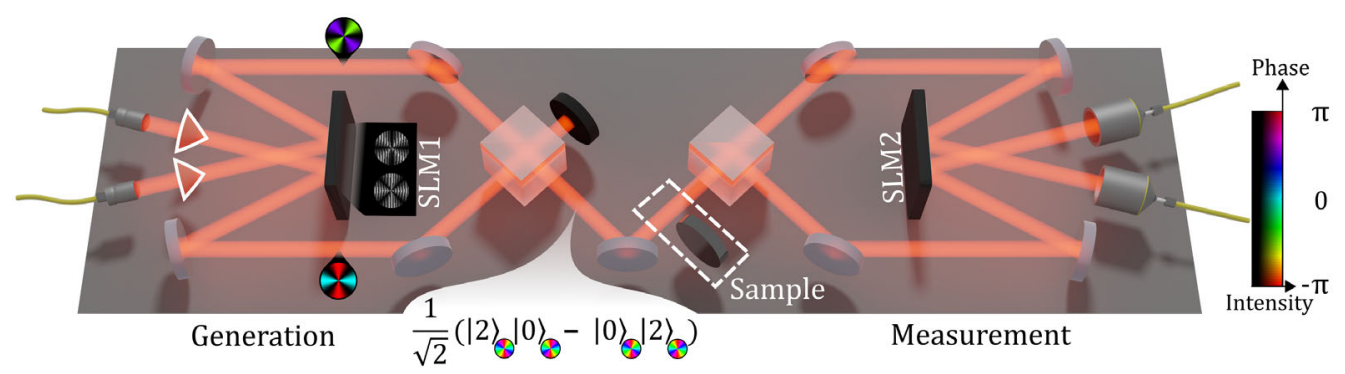

FIG. 1. Conceptual image of the experimental setup. Two holograms are used on the first SLM (SLM1) to imprint the wanted structures onto each photon, independently. The two photons are then overlapped using a beam splitter, to enable photon bunching into the same spatial structure, hence, allowing for a single beam operation when probing a sample. To measure the two-photon state, the photons are separated with a beam splitter and sent to SLM2 where another set of holograms are used to measure the structure of each photon (see main text for details). The insets show an example of the holograms displayed on SLM1 to generate an OAM N00N state with $\ell= \pm 2$, an example of a sample position, and the transverse field structures the photons have at different points of the setup, visualized by a two-dimensional colormap (see color bar on right). 
by projecting the photons on orthogonal petal structures, which can only result in an interference curve with perfect visibility in the case of bunching. Interestingly, projecting both photons on identical petal structures can produce a perfect fringe visibility irrespective of bunching, although with an increased amplitude in the case of bunching (see Supplemental Material [18]).

Since rotating the light field by an angle $\varphi$ induces a phase $e^{i \ell N \varphi}$ on an N-photon Fock state [10], the theoretically expected optimal detection rate is $\langle\hat{M}\rangle=(M / 2)$ $[1-\cos (2 N \ell \varphi)]$, where $M$ is the number of repetitions of the measurement and $N$ is the number of photons used in the N00N state. From the detection rate, the theoretical scaling of the angular uncertainty can be expressed as

$$
\Delta \varphi=\frac{\langle\Delta \hat{M}\rangle}{|\partial\langle\hat{M}\rangle / \partial \varphi|}=\frac{1}{2 \sqrt{M} N \ell},
$$

which saturates the quantum Cramér-Rao bound. For derivations of Eq. (1) and the bound, based on Refs. [10,2830], see Supplemental Material [18].

The rotation measurements with $\ell=\{ \pm 1, \pm 10, \pm 100\}$ are shown in Fig. 2. The figure shows that increasing the amount of OAM increases the achievable resolution, and changing from a single-photon to a two-photon NOON state doubles the resolution.

To further analyze the measured data, we estimate the Fisher information and angular precision for each measurement. Therefore, we first fit a curve to each set of the measured data using a weighted nonlinear least squares fit (each point is weighted by the reciprocal of the measured variance). The fitted curve is

$$
\frac{A}{2}\left[1-\cos \left(2 N \ell \frac{\pi}{180^{\circ}} \varphi-c\right)\right]+D,
$$

where $A$ is the amplitude of the cosine curve, $D$ is the offset, and $c$ sets the position of $0^{\circ}$ rotation. Hence, $[A /(A+2 D)]$ gives an estimate of the visibility of the curve, based on the fit. For the single-photon measurements, we obtain an average visibility of 0.999 , whereas for the two-photon measurements the corresponding value is 0.956 averaged over all measurements. The maximum standard error for the visibilities is 0.011 , calculated for the two-photon $\ell= \pm 100$ state, from the confidence intervals of the fitting parameters.

From these fits and the estimated system efficiencies (around 0.015 for a single photon with $\ell=1$ and 0.002 when $\ell=100$ ) we are able to derive the expected Fisher information (see Supplemental Material [18]). We also calculate the angular uncertainty using

$$
\Delta \varphi=\frac{\Delta M(\varphi)}{A N \ell \frac{\pi}{180^{\circ}}\left|\sin \left(2 N \ell \frac{\pi}{180^{\circ}} \varphi-c\right)\right|},
$$

where $\Delta M(\varphi)$ is the standard deviation for each measurement angle calculated from around 25 repetitions,
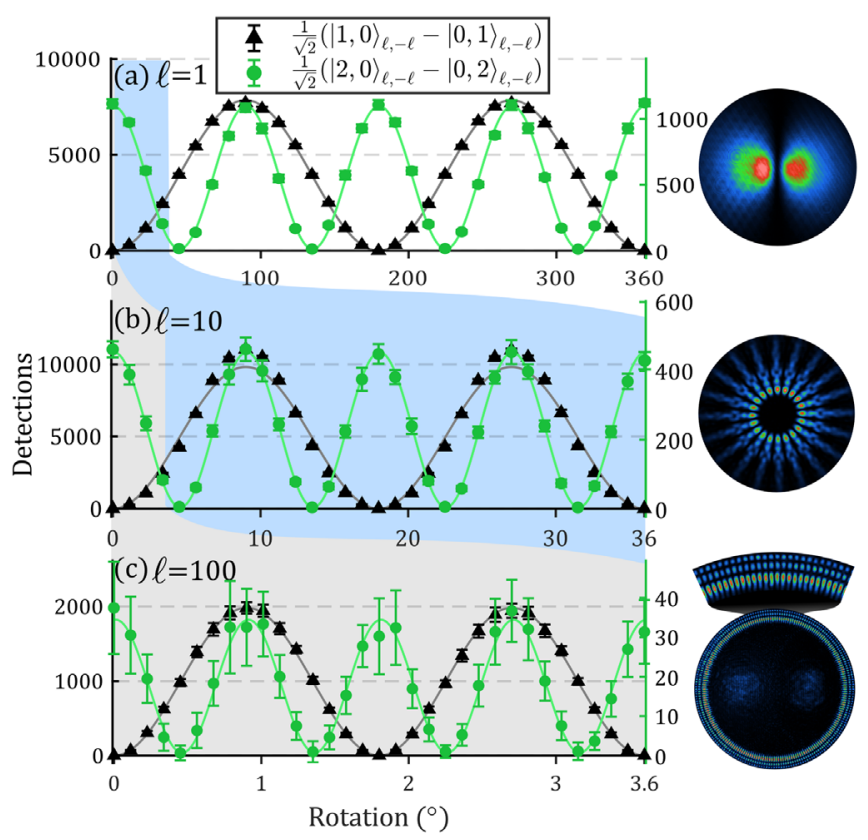

FIG. 2. Detected single photons and two-photon coincidences as a function of rotation angle. The single photons were prepared in the modes shown in the insets (insets show false-color images of structures taken with camera and laser light), and the corresponding two-photon N00N states were created by imprinting the same structure on one photon and its orthogonal pair (same structure rotated by $180^{\circ} / 2 \ell$ ) on the other. The plots in (a)(c) show single photon (two-photon) counts within integration

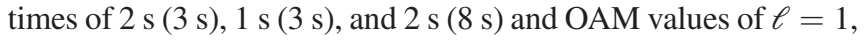
10 , and 100, respectively. The error bars have been calculated as standard deviations from at least 19 repetitions of the measurement at each point, and the solid lines are fits of the form shown in Eq. (2). The decreased period between oscillations shows the angular superresolution achieved with the two-photon NOON states [31]. For the two-photon measurements, accidental coincidences have been subtracted.

depending on the photon number and OAM value. The Fisher information and angular precision are shown in Fig. 3 for $\ell=100$ and in the Supplemental Material [18] for $\ell=1$.

Plots (a) and (c) in Fig. 3 show that the expected Fisher information curves follow the reciprocal of the rotation angle variance, meaning that the results are close to the Cramér-Rao bound of our specific state measurement [4]. Similarly, the expected angular uncertainties mostly agree with the measured angular uncertainties. This indicates that the achieved precision is close to the maximum precision bounded by Poissonian noise. The differences between the expected curves and measured data stem from the limited number of repetitions used to calculate the standard deviation, the decoupling of the system during long measurements, and differences in system losses due to different bandwidths of our single-photon source and the laser used in characterization. The change in precision 


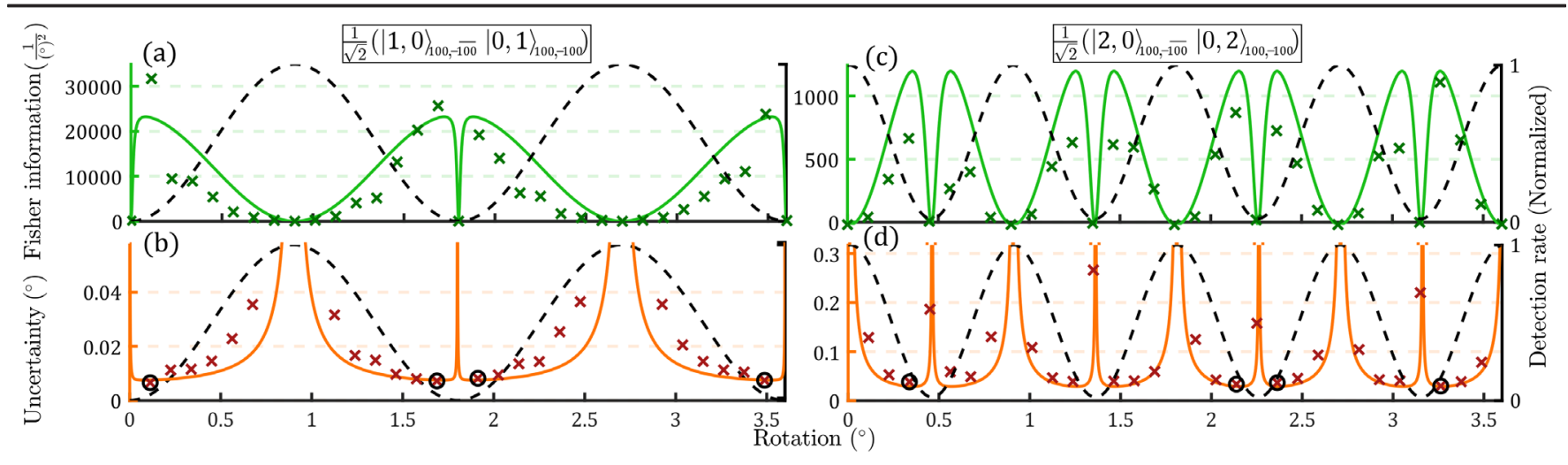

FIG. 3. Fisher information and angular uncertainty for $\ell=100$ N00N states. On the upper row, the continuous green line is the Fisher information multiplied by the estimate for the total number of heralded single photons (or photon pairs) before losses. The green crosses are the reciprocal of the variance calculated from Eq. (3). On the bottom row, the continuous curves are calculated using Eq. (3) and Poissonian errors calculated from the fit. The red crosses are the experimentally determined uncertainties, calculated using Eq. (3). Plots (a) and (b) display the heralded single-photon data, and (c) and (d) contain two-photon data. In all graphs, the black dashed lines depict the interference curves for reference. On the bottom row, the uncertainty values that have been circled are used for calculating the respective sensitivities in Fig. 4.

caused by errors that were larger than Poissonian is especially apparent with the $\ell=100$ measurements where a small drift over time has a comparatively large effect on the alignment of the small structures of the transverse field.

Finally, we compare the sensitivities that are achievable with two-photon NOON states to single-photon sensitivities, using different values of OAM. Figures 3(c) and 3(d) show that the best angular precision tends to be found at the same values of $\varphi$ where the Fisher information is maximized. Therefore, to quantify the achievable sensitivities with different values of $N$ and $\ell$, we take the four smallest values of angular uncertainty $\Delta \varphi$ from each measurement, close to the point of maximum Fisher information. We then calculate the reciprocal for each of these values and define it as the sensitivity. To be able to compare sensitivities between different measurements, we normalize them by dividing each value $\Delta \varphi$ by $(\sqrt{A+D} / A)$, which removes the dependence of $\Delta \varphi$ on the varying number of detections in each measurement. These values are plotted in Fig. 4, along with theoretically expected maximum sensitivities for the measurement scheme used.

Fig. 4 shows that the measured two-photon states are more sensitive than their one-photon counterparts, although the theoretical scaling was not reached with two-photon states with large OAM. This discrepancy is caused by the nonperfect visibilities of the measured interference curves, in addition to the increasing complexity of the structures and their decreasing efficiencies, causing the alignment to be more sensitive while requiring longer measuring times. As a result, a slow misalignment over time has a larger effect on the variability of detection rates over the repeated measurements.

In the presented experiment, we created twisted oneand two-photon NOON states and verified the scaling they enable for angular resolution and sensitivity, when increasing the photon number or OAM. In order to verify these properties, we rotated the measuring hologram on a SLM to simulate a rotation of the light field. Hence, the method could be directly applied to precisely aligning two rotational reference frames, e.g., in a communication channel [13]. However, in order to apply the method for

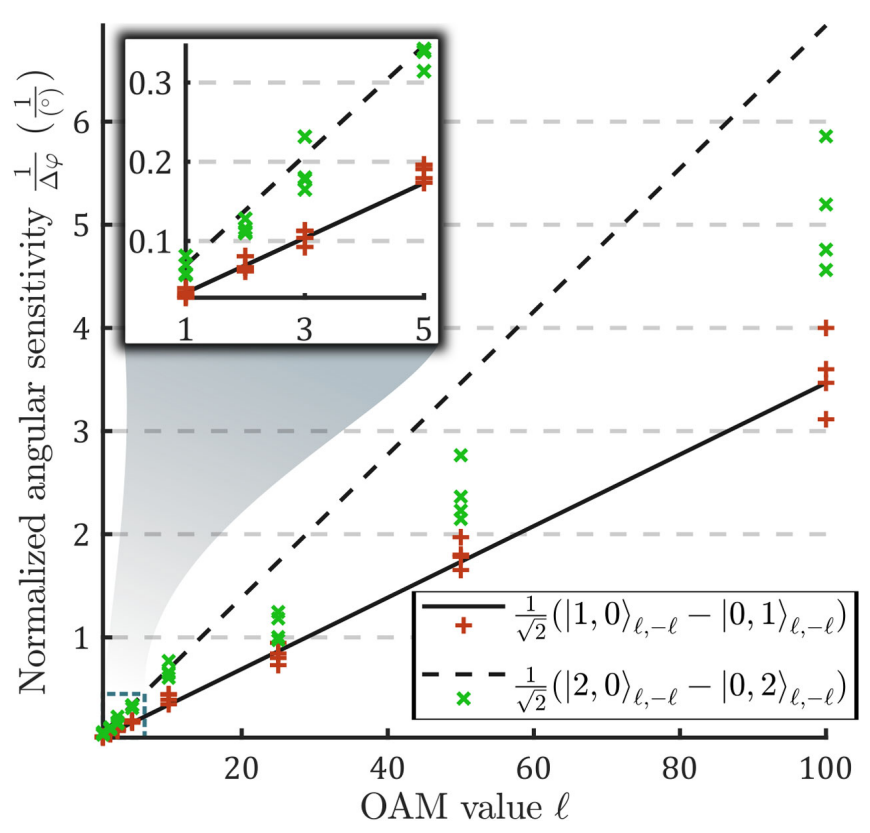

FIG. 4. Measurement sensitivities of single-photon and twophoton N00N states. The theoretical curves are calculated using Poissonian errors and a visibility of 0.9999 for the cosine curve introduced in Eq. (2). The crosses represent the four normalized sensitivities calculated from the uncertainty values chosen from each measurement. The mostly linear dependence of $1 / \Delta \varphi$ on OAM follows the scaling of angular uncertainty introduced in Eq. (1). 
measuring rotations caused by a separate system, the probed sample needs to be the one providing an OAM-dependent phase onto our two-photon state. This could be achieved by embedding an image rotator (e.g., a Dove prism) into the object whose rotation we want to measure, or by probing samples that interact with the N00N state by inducing an OAM-dependent phase which is contingent upon some property of the sample. Hence, the scheme is not restricted to only measuring rotations of a light field or reference frame, but can be used to measure any OAM-dependent phase changes. Additionally, since adding photons into the NOON state can be done irrespective of the aperture of the system, the increased angular resolution provided by a NOON state might be beneficial in tasks with a limited aperture size. However, in order to push the limits of achievable sensitivity with this measurement scheme, the system losses need to be reduced and a more appropriate estimator for the rotation angle should be devised [5,32].

In summary, we showed that by structuring and overlaying two photons, a high-fidelity two-photon N00N state can be created between any two high-OAM spatial structures. With this method, we are able to bunch two photons into modes with up to OAM $100 \hbar$, with minimal experimental complexity. For future implementations, improving the method's efficiency would be key in pushing the achievable sensitivity. The current losses are caused by the methods used for generation and detection, as well as the probabilistic overlapping and separation of the photon pair. To show a quantum advantage over a lossless classical system with perfect visibility, the condition $\eta^{N} V^{2} N>1$, introduced in references [5,31], needs to be met. With the average visibility $V=0.956$ for our two-photon rotation sensing, the single-photon efficiency needs to be $\eta>0.74$ to overcome the shot-noise limit. However, the efficiency of the system could be increased by using methods that are, in principle, lossless for preparing and measuring the spatial modes [33], and for combining the two photons into the same beam path $[34,35]$.

The authors thank Shashi Prabhakar, Rafael F. Barros, and Lea Kopf for fruitful discussions. M. H. and R.F. acknowledge the support of the Academy of Finland through the Competitive Funding to Strengthen University Research Profiles (Decision 301820) (Grant No. 308596), and the Photonics Research and Innovation Flagship (PREIN_Decision 320165). M. H. also acknowledges support from the Doctoral School of Tampere University and the Magnus Ehrnrooth Foundation through its graduate student scholarship. F. B. acknowledges support from the National Research Council's High Throughput Secure Networks challenge program and the Joint Centre for Extreme Photonics. R. F. also acknowledges support from the Academy of Finland through the Academy Research Fellowship (Decision 332399). *markus.hiekkamaki@tuni.fi

†obert.fickler@tuni.fi

[1] J. G. Rarity, P. R. Tapster, E. Jakeman, T. Larchuk, R. A. Campos, M.C. Teich, and B.E. A. Saleh, Two-Photon Interference in a Mach-Zehnder Interferometer, Phys. Rev. Lett. 65, 1348 (1990).

[2] A. Kuzmich and L. Mandel, Sub-shot-noise interferometric measurements with two-photon states, Quantum Semiclassical Opt. 10, 493 (1998).

[3] J. P. Dowling, Quantum optical metrology-the lowdown on high-N00N states, Contemp. Phys. 49, 125 (2008).

[4] E. Polino, M. Valeri, N. Spagnolo, and F. Sciarrino, Photonic quantum metrology, AVS Quantum Sci. 2, 024703 (2020).

[5] S. Slussarenko, M. M. Weston, H. M. Chrzanowski, L. K. Shalm, V. B. Verma, S. Woo Nam, and G. J. Pryde, Unconditional violation of the shot-noise limit in photonic quantum metrology, Nat. Photonics 11, 700 (2017).

[6] S. M. Barnett and R. Zambrini, Resolution in rotation measurements, J. Mod. Opt. 53, 613 (2006).

[7] M. Erhard, R. Fickler, M. Krenn, and A. Zeilinger, Twisted photons: New quantum perspectives in high dimensions, Light Sci. Appl. 7, 17146 (2018).

[8] R. Fickler, G. Campbell, B. Buchler, P. Koy Lam, and A. Zeilinger, Quantum entanglement of angular momentum states with quantum numbers up to 10,010, Proc. Natl. Acad. Sci. U.S.A. 113, 13642 (2016).

[9] M. J. Padgett, F. M. Miatto, M. P. J. Lavery, A. Zeilinger, and R.W. Boyd, Divergence of an orbital-angularmomentum-carrying beam upon propagation, New J. Phys. 17, 023011 (2015).

[10] A. Kumar Jha, G. S. Agarwal, and R. W. Boyd, Supersensitive measurement of angular displacements using entangled photons, Phys. Rev. A 83, 053829 (2011).

[11] R. Fickler, R. Lapkiewicz, W. N. Plick, M. Krenn, C. Schaeff, S. Ramelow, and A. Zeilinger, Quantum entanglement of high angular momenta, Science 338, 640 (2012).

[12] K. Liu, C. Cai, J. Li, L. Ma, H. Sun, and J. Gao, Squeezingenhanced rotating-angle measurement beyond the quantum limit, Appl. Phys. Lett. 113, 261103 (2018).

[13] V. D’ambrosio, N. Spagnolo, L. Del Re, S. Slussarenko, Y. Li, L. Chuan Kwek, L. Marrucci, S. P. Walborn, L. Aolita, and F. Sciarrino, Photonic polarization gears for ultra-sensitive angular measurements, Nat. Commun. 4, 1 (2013).

[14] Y. Ming, J. Tang, Z.-x. Chen, F. Xu, L.-j. Zhang, and Y.-q. $\mathrm{Lu}$, Generation of NOON state with orbital angular momentum in a twisted nonlinear photonic crystal, IEEE J. Sel. Top. Quantum Electron. 21, 225 (2014).

[15] M. Hiekkamäki and R. Fickler, High-Dimensional TwoPhoton Interference Effects in Spatial Modes, Phys. Rev. Lett. 126, 123601 (2021).

[16] C. K. Hong, Z. Y. Ou, and L. Mandel, Measurement of Subpicosecond Time Intervals Between Two Photons by Interference, Phys. Rev. Lett. 59, 2044 (1987).

[17] A. Datta, L. Zhang, N. Thomas-Peter, U. Dorner, B. J. Smith, and I. A. Walmsley, Quantum metrology with imperfect states and detectors, Phys. Rev. A 83, 063836 (2011). 
[18] See Supplemental Material at http://link.aps.org/supplemental/ 10.1103/PhysRevLett.127.263601 for detailed derivations, additional data, and a detailed description of the experimental setup.

[19] E. Bolduc, N. Bent, E. Santamato, E. Karimi, and R. W. Boyd, Exact solution to simultaneous intensity and phase encryption with a single phase-only hologram, Opt. Lett. 38, 3546 (2013).

[20] A. Forbes, A. Dudley, and M. McLaren, Creation and detection of optical modes with spatial light modulators, Adv. Opt. Photonics 8, 200 (2016).

[21] F. Bouchard, N. Herrera Valencia, F. Brandt, R. Fickler, M. Huber, and M. Malik, Measuring azimuthal and radial modes of photons, Opt. Express 26, 31925 (2018).

[22] A. Mair, A. Vaziri, G. Weihs, and A. Zeilinger, Entanglement of the orbital angular momentum states of photons, Nature (London) 412, 313 (2001).

[23] A. Jesacher, A. Schwaighofer, S. Fürhapter, C. Maurer, S. Bernet, and M. Ritsch-Marte, Wavefront correction of spatial light modulators using an optical vortex image, Opt. Express 15, 5801 (2007).

[24] C. K. Hong and L. Mandel, Experimental Realization of a Localized One-Photon State, Phys. Rev. Lett. 56, 58 (1986).

[25] O. Gühne and G. Tóth, Entanglement detection, Phys. Rep. 474, 1 (2009).

[26] R. Fickler, R. Lapkiewicz, S. Ramelow, and A. Zeilinger, Quantum entanglement of complex photon polarization patterns in vector beams, Phys. Rev. A 89, 060301(R) (2014).
[27] I. A Litvin, S. Ngcobo, D. Naidoo, K. Ait-Ameur, and A. Forbes, Doughnut laser beam as an incoherent superposition of two petal beams, Opt. Lett. 39, 704 (2014).

[28] M. O. Scully and J. P. Dowling, Quantum-noise limits to matter-wave interferometry, Phys. Rev. A 48, 3186 (1993).

[29] R. Demkowicz-Dobrzański, M. Jarzyna, and J. Kołodyński, Quantum limits in optical interferometry, Prog. Opt. 60, 345 (2015).

[30] G. Grynberg, A. Aspect, and C. Fabre, Introduction to Quantum Optics: From the Semi-Classical Approach to Quantized Light (Cambridge University Press, Cambridge, England, 2010), Chap. 4B, pp. 332-333.

[31] K. J. Resch, K. L. Pregnell, R. Prevedel, A. Gilchrist, G. J. Pryde, J. L. O'Brien, and A. G. White, Time-Reversal and Super-Resolving Phase Measurements, Phys. Rev. Lett. 98, 223601 (2007).

[32] A. Lyons, G. C. Knee, E. Bolduc, T. Roger, J. Leach, E. M. Gauger, and D. Faccio, Attosecond-resolution HongOu-Mandel interferometry, Sci. Adv. 4, eaap9416 (2018).

[33] M. Hiekkamäki, S. Prabhakar, and R. Fickler, Near-perfect measuring of full-field transverse-spatial modes of light, Opt. Express 27, 31456 (2019).

[34] G. Labroille, B. Denolle, P. Jian, P. Genevaux, N. Treps, and J.-F. Morizur, Efficient and mode selective spatial mode multiplexer based on multi-plane light conversion, Opt. Express 22, 15599 (2014).

[35] N. K. Fontaine, R. Ryf, H. Chen, D. T. Neilson, K. Kim, and J. Carpenter, Laguerre-Gaussian mode sorter, Nat. Commun. 10, 1865 (2019). 